\title{
Application of Analog Network Coding to MIMO Two-Way Relay Channel in Cellular Systems
}

\author{
Ming Gan, Zhiguo Ding, Member, IEEE, Xuchu Dai
}

\begin{abstract}
An efficient analog network coding transmission protocol is proposed in this letter for a MIMO two way cellular network. Block signal alignment is first proposed to null the inter-user interference for multi-antenna users, which makes the dimensions of aligned space larger compared with the existing signal alignment. Two algorithms are developed to jointly design the precoding matrices at the relay and BS for outage optimization. Especially, the last algorithm is designed to maximize the effective channel gain to the effective noise gain ratio. The performance of this transmission protocol is also verified by simulations.
\end{abstract}

Index Terms-MIMO two-way, beamforming, block signal alignment, outage optimization.

\section{INTRODUCTION}

Multiuser-MIMO two-way relaying technique has emerged as a promising technique to improve the spectrum efficiency by applying multi-antenna techniques to eliminate inter-user interference [1]. To further reduce the requirement of node antennas, the concept of signal alignment has been applied to two-way relaying networks [2]. Most existing works about multiuser two-way networks are to design precoding matrices by completely diagonalizing the channel matrices. Compared to the concept of block diagonalization, it has been recognized that perfect diagonalization results in some performance loss [3]. Therefore, in this letter we will develop a coordinated beamforming scheme to null the inter-user interference using a new approach of block signal alignment (BSA), and derive closed-form expressions for the beamforming matrices based on orthogonal projection. This new scheme is completely different from [2] whose key idea was point to point signal alignment (P2PSA) realized by perfect diagonalization. BSA makes the dimensions of aligned space larger compared with P2PSA such that it achieves better performance both in outage probability and ergodic capacity for multi-antenna users.

Focusing on outage optimization for each user and applying the beamforming structures designed at the relay and base station (BS), we first develop one algorithm to jointly design the beamforming matrices at the relay and BS based on MMSE and Algebraic Norm-Maximizing (ANOMAX) [4]. Then we develop another algorithm based on Algebraic NormMaximizing without Normalization (ANMwoN), which is first proposed in this letter to maximize the Frobenius norms of the effective channels without normalizing beamforming matrices as in ANOMAX. Instead of only maximizing the effective channel gain in ANOMAX, the effective channel gain to the effective noise gain ratio (ECG2ENGR) is maximized for the relay precoder. Simulation results demonstrate the performance of the proposed transmission approaches.

M. Gan and X. Dai are with Department of Electronic Engineering and Information Science, University of Science and Technology of China, P.R.China. Z. Ding is with the School of Electrical, Electronic and Computer Engineering, Newcastle University, UK.

\section{Description of the Protocol}

Consider a MIMO cellular network with $M$ mobile users, one BS and a single relay, where each node operates in half-duplex. Both the relay and the BS are equipped with $N$ antennas. The $m$-th mobile user has $K_{m}$ antennas, for $m=1, \ldots, M$. The total number of antennas at all mobile users is defined to be $K=\sum_{m=1}^{M} K_{m}$. All wireless channels are assumed to be Rayleigh fading, reciprocal, and constant over two transmission phases and there are no direct links between the BS and mobile users due to severe shadowing. It is assumed that the BS and relay have global channel state information (CSI) prior to transmission.

Without loss of generality, we focus on the $m$-th user and the similar operations for other users. To achieve full multiplexing gain, it is assumed that each node transmits full data streams, equal to the number of its antennas. During the multiple access phase, the BS transmits the precoded symbols, Ps, where $\mathbf{s}=\left[\begin{array}{lll}\mathbf{s}_{1}^{T} & \cdots & \mathbf{s}_{M}^{T}\end{array}\right]^{T}, \mathbf{s}_{m} \in \mathbb{C}^{K_{m} \times 1}$ denotes the message to the $m$-th user with unit power and $\mathbf{P}$ is a $N \times K$ precoding matrix. It is assumed that the transmission power for the $m$-th user at the BS is $\mathbf{P}_{m}^{o w}$, i.e., $\operatorname{tr}\left\{\mathbf{P} \mathbf{P}^{H}\right\} \leq \sum_{m=1}^{M} \mathbf{P}_{m}^{o w}$, where $\operatorname{tr}\{\cdot\}$ denotes the trace. Meanwhile, the $m$-th users sends its message vector $\mathbf{s}_{m}^{\prime} \in \mathbb{C}^{K_{m} \times 1}$ with unit power to the relay. Therefore, the observations at the relay are given as

$$
\mathbf{r}=\mathbf{G P s}+\sum_{m=1}^{M} \mathbf{H}_{m} \mathbf{s}_{m}^{\prime}+\mathbf{n}_{R}
$$

where $\mathbf{G} \in \mathbb{C}^{N \times N}\left(\mathbf{H}_{m} \in \mathbb{C}^{N \times K_{m}}\right)$ is the full-rank channel matrix from the $\mathrm{BS}$ (the $m$-th user) to the relay, $\mathbf{n}_{R} \in \mathbb{C}^{N \times 1}$ denotes additive white Gaussian noise vector whose components follow $\mathcal{C N}\left(0, \sigma_{r}^{2}\right)$. During the broadcast phase, upon receiving $\mathbf{r}$, the relay amplifies it using the precoding matrix $\mathbf{W}$ and broadcasts the output of the precoder. The transmission power constraint for $m$-th user at the relay is $\mathrm{P}_{R_{m}}^{o w}$. Then the received signals at the $\mathrm{BS}$ can be written as

$$
\mathbf{y}_{B S}=\mathbf{G}^{H} \mathbf{W}\left(\mathbf{G P s}+\mathbf{H s}^{\prime}+\mathbf{n}_{R}\right)+\mathbf{n}_{B S},
$$

where $\mathbf{H}=\left[\begin{array}{lll}\mathbf{H}_{1} & \cdots & \mathbf{H}_{M}\end{array}\right]$ and $\mathbf{s}^{\prime}=\left[\begin{array}{lll}\mathbf{s}_{1}^{\prime} & \cdots & \mathbf{s}_{M}^{\prime T}\end{array}\right]^{T}$, and at the $m$-th user, the signal model can be expressed as

$$
\mathbf{y}_{m}=\mathbf{H}_{m}^{H} \mathbf{W}\left(\mathbf{G P s}+\mathbf{H s}^{\prime}+\mathbf{n}_{R}\right)+\mathbf{n}_{m},
$$

where $\mathbf{n}_{m} \in \mathbb{C}^{K_{m} \times 1}$ and $\mathbf{n}_{B S} \in \mathbb{C}^{N \times 1}$ are defined similarly to $\mathbf{n}_{R}$, whose components follow $\mathcal{C N}\left(0, \sigma_{m}^{2}\right)$ and $\mathcal{C N}\left(0, \sigma_{B S}^{2}\right)$, respectively. Observe that each user receives a mixture of desirable signals, self-interference (SI), interference from the messages sent from and to other users and amplified noise, where SI can be easily removed with the CSI and the last two terms will severely degrade the performance. 


\section{The Precoding Design at the BS and Relay}

Here we consider the situation of $N=K 1$ Instead of P2PSA proposed in [2], the concept of block diagonalization is applied for the coordinated beamforming design, and the key idea of this design is BSA that the observations from and to the same mobile user can lie in the same space, i.e., $\mathbf{s}_{m}$ and $\mathbf{s}_{m}^{\prime}$ aligned together. The dimensions of aligned space become larger since the two coefficient matrices before the two grouped signal vectors are different, not the identity matrices, after the coordinated beamforming design. This can be facilitated by defining the precoder $\mathbf{P}$ at the BS to meet

$$
\mathbf{H}^{-1} \mathbf{G P}=\operatorname{diag}\left(\left[\begin{array}{lll}
\mathbf{A}_{1} & \cdots & \mathbf{A}_{M}
\end{array}\right]\right),
$$

where $\mathbf{P}=\left[\begin{array}{llll}\mathbf{P}_{1} & \mathbf{P}_{2} & \cdots & \mathbf{P}_{M}\end{array}\right], \operatorname{diag}()$ represents the block diagonal matrix, and $\mathbf{A}_{m} \in \mathbb{C}^{K_{m} \times K_{m}}, m=1,2, \ldots, M$ , is a full rank matrix. The use of the precoding matrix defined in (4) can convert the signal model in (1) to $\mathbf{r}=\sum_{m=1}^{M} \mathbf{H}_{m}\left(\mathbf{A}_{m} \mathbf{s}_{m}+\mathbf{s}_{m}^{\prime}\right)+\mathbf{n}_{R}$, where the user signals and the signals from the BS are aligned together. The existing scheme in [2] is a special case by letting all $\mathbf{A}_{m}$ to be identity matrices. To satisfy the condition (4), the preorder $\mathbf{P}$ can be obtained by using orthogonal projection method. First, define $\mathbf{G}^{\prime}=\mathbf{H}^{-1} \mathbf{G}$ and represent $\mathbf{G}^{\prime}=\left[\begin{array}{lll}\mathbf{G}_{1}^{\prime T} & \cdots & \mathbf{G}_{M}^{\prime T}\end{array}\right]^{T}$. Then using the orthogonal projection matrix $\mathbf{Q}_{m}=\left(\mathbf{I}_{N}-\tilde{\mathbf{G}}_{m}^{\prime H}\left(\tilde{\mathbf{G}}_{m}^{\prime} \tilde{\mathbf{G}}_{m}^{\prime H}\right)^{-1} \tilde{\mathbf{G}}_{m}^{\prime}\right)$ where $\tilde{\mathbf{G}}_{m}^{\prime}=\left[\begin{array}{llllll}\mathbf{G}_{1}^{\prime T} & \cdots & \mathbf{G}_{m-1}^{\prime T} & \mathbf{G}_{m+1}^{\prime T} & \cdots & \mathbf{G}_{M}^{\prime T}\end{array}\right]^{T} \in$ $\mathbb{C}^{\left(K-K_{m}\right) \times N}$, the precoder's component matrix $\mathbf{P}_{m}$ can be expressed as

$$
\mathbf{P}_{m}=\mathbf{Q}_{m} \mathbf{G}_{m}^{\prime H} \mathbf{D}_{m},
$$

where the fact $\mathbf{G}_{i}^{\prime} \mathbf{Q}_{j}=\mathbf{0}$ for $i \neq j$ is used and $\mathbf{D}_{m}$ is used to meet the power constraint for the $m$-th user at the $\mathrm{BS}$ as

$$
\mathbf{P}_{m}^{o w}=\operatorname{tr}\left\{\mathbf{P}_{m} \mathbf{P}_{m}^{H}\right\}=\operatorname{tr}\left\{\mathbf{T}_{m} \mathbf{D}_{m} \mathbf{D}_{m}^{H}\right\},
$$

where $\mathbf{T}_{m}=\mathbf{G}_{m}^{\prime} \mathbf{Q}_{m} \mathbf{G}_{m}^{\prime H}$, a $K_{m} \times K_{m}$ matrix. With the Hermitian matrix assumption for the power constraint matrix $\mathbf{D}_{m}$ in (6), it can be computed via

$$
\mathbf{D}_{m}=\frac{\mathbf{P}_{m}^{o w}}{\sqrt{K_{m}}}\left(\mathbf{T}_{m}\right)^{-\frac{1}{2}},
$$

which yields a deterministic solution for the precoding matrices. Obviously such a fixed solution is not optimal, and how to optimize precoding will be discussed in the next section. Substituting (4) into (3), we obtain $\mathbf{y}_{m}=$ $\mathbf{H}_{m}^{H} \mathbf{W}\left(\mathbf{H}\left(\operatorname{diag}\left(\left[\begin{array}{lll}\mathbf{T}_{1} \mathbf{D}_{1} & \cdots & \mathbf{T}_{M} \mathbf{D}_{M}\end{array}\right]\right) \mathbf{s}+\mathbf{s}^{\prime}\right)+\mathbf{n}_{R}\right)+$

\footnotetext{
${ }^{1}$ The proposed approach can be extended to the scenarios with $N \leq K$ as follows. The precoding design will be accomplished in two phases. The strategy introduced in Section III and IV will be carried out first, and then a further step is to optimize the user precoding matrices. The second step is analogous to antenna selection in traditional MIMO systems. Specifically, the message vector $\mathbf{s}_{m}^{\prime} \in \mathbb{C}^{K_{m} \times 1}$ sent by the $m$-th user denotes $\mathbf{s}^{\prime}{ }_{m}=\mathbf{F}_{m} \mathbf{x}^{\prime}{ }_{m}$, where the precoding matrix $\mathbf{F}_{m} \in \mathbb{C}^{K_{m} \times K_{m}^{\prime}}$ can be set as the $K_{m}^{\prime}$ right singular vectors of $H_{m}$ or the end to end effective channel matrix corresponding to the largest singular values, and $\mathbf{x}_{m}^{\prime} \in \mathbb{C}^{K_{m}^{\prime} \times 1}$ is the coded signal. The parameter $K^{\prime}=\Sigma_{m=1}^{M} K_{m}^{\prime}$ is chosen to ensure that the effective channel matrix between the relay and the users $\mathbf{H}^{\prime}=\left[\begin{array}{lll}\mathbf{H}_{1} \mathbf{F}_{1} & \cdots & \mathbf{H}_{M} \mathbf{F}_{M}\end{array}\right] \in \mathcal{C}^{N \times K^{\prime}}$ is non-singular, i.e. $\left(N=K^{\prime}\right)$, which is used instead of $\mathbf{H}$. The reader is referred to [5] for a more detailed discussion on the user precoding design.
}

$\mathbf{n}_{m}$. To remove the inter-user interference, the precoding matrix $\mathbf{W}$ at the relay should follow

$$
\mathbf{H}^{H} \mathbf{W H}=\operatorname{diag}\left(\left[\begin{array}{lll}
\mathbf{B}_{1} & \cdots & \mathbf{B}_{M}
\end{array}\right]\right),
$$

where $\mathbf{W}=\sum_{m=1}^{M} \mathbf{W}_{m}, \mathbf{B}_{m}$ is a full rank $K_{m} \times K_{m}$ matrix. Note that the condition (8) is not perfect diagonalization, enhancing the BSA. It can be realized by the combination of the orthogonal projection and eigen value decomposition (EVD). First define $\tilde{\mathbf{H}}_{m}=\left[\begin{array}{llllll}\mathbf{H}_{1} & \cdots & \mathbf{H}_{m-1} & \mathbf{H}_{m+1} & \cdots & \mathbf{H}_{M}\end{array}\right] \quad \in$ $\mathbb{C}^{N \times\left(K-K_{m}\right)} \quad$ with its orthogonal projection matrix $\quad$ is $\quad \mathbf{Q}_{m}^{\prime}=\left(\mathbf{I}_{N}-\tilde{\mathbf{H}}_{m}\left(\tilde{\mathbf{H}}_{m}^{H} \tilde{\mathbf{H}}_{m}\right)^{-1} \tilde{\mathbf{H}}_{m}^{H}\right)$ of idempotent property. Then using EVD, i.e., $\mathbf{Q}_{m}^{\prime}=\left[\begin{array}{ll}\mathbf{U}_{m_{1}} & \mathbf{U}_{m_{0}}\end{array}\right]\left[\begin{array}{cr}\boldsymbol{\Sigma}_{m} & 0 \\ 0 & 0\end{array}\right]\left[\begin{array}{ll}\mathbf{U}_{m_{1}} & \mathbf{U}_{m_{0}}\end{array}\right]^{H}$, where $\mathbf{U}_{m_{1}} \in \mathbb{C}^{N \times K_{m}}$, the precoder's component matrix $\mathbf{W}_{m}$ can be computed via

$$
\mathbf{W}_{m}=\mathbf{U}_{m_{1}} \mathbf{D}_{m}^{\prime} \mathbf{U}_{m_{1}}^{H},
$$

where the fact $\mathbf{U}_{i_{1}}^{H} \mathbf{H}_{j}=\mathbf{0}$ for $i \neq j$ is used and $\mathbf{D}^{\prime}{ }_{m}$ is to ensure the power constraint for messages transmission to the $m$-th user at the relay as

$$
\begin{aligned}
\mathbf{P}_{R_{m}}^{o w} & =\operatorname{tr}\left\{\mathbf{W}_{m} \mathbf{r r} \mathbf{r}^{H} \mathbf{W}_{m}^{H}\right\} \\
& \approx \operatorname{tr}\left\{\mathbf{D}_{m}^{\prime H} \mathbf{D}_{m}^{\prime} \overline{\mathbf{T}}_{m}\left(\mathbf{T}_{m} \mathbf{D}_{m} \mathbf{D}_{m}^{H} \mathbf{T}_{m}^{H}+\mathbf{I}_{K_{m}}\right) \overline{\mathbf{T}}_{m}^{H}\right\},
\end{aligned}
$$

where $\overline{\mathbf{T}}_{m}=\mathbf{U}_{m_{1}}^{H} \mathbf{H}_{m}$ and the last approximation is due to the high signal-to-noise ratio (SNR) assumption. With the Hermitian matrix assumption for $\mathbf{D}^{\prime}{ }_{m}$, a deterministic solution of (10) can be expressed as

$$
\mathbf{D}_{m}^{\prime}=\frac{\mathbf{P}_{R_{m}}^{o w}}{\sqrt{K_{m}}}\left(\overline{\mathbf{T}}_{m}\left(\mathbf{T}_{m} \mathbf{D}_{m} \mathbf{D}_{m}^{H} \mathbf{T}_{m}^{H}+\mathbf{I}_{K_{m}}\right) \overline{\mathbf{T}}_{m}^{H}\right)^{-\frac{1}{2}} .
$$

In the second phase, the relay nulls the inter-user interference using the fact $\mathbf{W}_{i} \mathbf{H}_{j}=\mathbf{0}$ and $\mathbf{H}_{i}^{H} \mathbf{W}_{j}=\mathbf{0}$, for $i \neq j$. Hence (3) can be rewritten as

$$
\begin{aligned}
\mathbf{y}_{m} & =\mathbf{B}_{m} \mathbf{A}_{m} \mathbf{s}_{m}+\mathbf{B}_{m} \mathbf{s}^{\prime}{ }_{m}+\mathbf{H}_{m}^{H} \mathbf{W}_{m} \mathbf{n}_{R}+\mathbf{n}_{m} \\
& =\overline{\mathbf{T}}_{m}^{H} \mathbf{D}^{\prime}{ }_{m} \overline{\mathbf{T}}_{m} \mathbf{T}_{m} \mathbf{D}_{m} \mathbf{s}_{m}+\overline{\mathbf{T}}_{m}^{H} \mathbf{D}^{\prime}{ }_{m} \overline{\mathbf{T}}_{m} \mathbf{s}^{\prime}{ }_{m}+\tilde{\mathbf{n}}_{m},
\end{aligned}
$$

where $\mathbf{B}_{m}=\overline{\mathbf{T}}_{m}^{H} \mathbf{D}_{m}^{\prime} \overline{\mathbf{T}}_{m}, \mathbf{A}_{m}=\mathbf{T}_{m} \mathbf{D}_{m}$ and $\tilde{\mathbf{n}}_{m}=$ $\overline{\mathbf{T}}_{m}^{H} \mathbf{D}_{m}^{\prime} \mathbf{U}_{m_{1}}^{H} \mathbf{n}_{R}+\mathbf{n}_{m}$. As seen from (12), the $m$-th user only observes a mixture of the desired signals, SI and enhanced noise, where the signals from and to other users have been eliminated completely because of the careful precoding design at the BS and relay. After the cancellation of SI, the mutual information at the $m$-th user and BS can be calculated by

$$
\mathcal{I}_{m}=\log \operatorname{det}\left(\mathbf{I}_{K_{m}}+\mathbf{F}_{m} \mathbf{F}_{m}^{H} \tilde{\mathbf{R}}_{m}^{-1}\right),
$$

where $\mathbf{F}_{m}=\overline{\mathbf{T}}_{m}^{H} \mathbf{D}_{m}^{\prime} \overline{\mathbf{T}}_{m} \mathbf{T}_{m} \mathbf{D}_{m} \quad$ and $\quad \tilde{\mathbf{R}}_{m}=$ $\overline{\mathbf{T}}_{m}^{H} \mathbf{D}_{m}^{\prime} \mathbf{D}_{m}^{\prime H} \overline{\mathbf{T}}_{m} \sigma_{r}^{2}+\sigma_{m}^{2} \mathbf{I}_{K_{m}}$.

\section{Outage Optimization FOR EACH USER}

Due to the poor capability of users who are equipped with less antennas, we focus on the precoding design optimization for users. Based on the beamforming structures designed in Section III, we jointly determine the optimal power normalization matrices $\mathbf{D}_{m}$ and $\mathbf{D}_{m}^{\prime}$ for the $m$-th user's 
outage optimization without Hermitian matrices assumption, i.e., $\min _{\mathbf{D}_{m}, \mathbf{D}^{\prime} m} \operatorname{Pro}\left(\mathcal{I}_{m}<2 K_{m} R\right)$, where $R$ is the target data rate per channel use. However, this problem is extremely complicated, non-linear and non-convex and hence difficult to be solved. To obtain the approximate optimal solution, we propose two iterative algorithms based on alternating optimization that updates one precoder at a time while fixing another. It is assumed that the power constraints for the $m$-th user at both BS and relay are 1 .

\section{A. Outage Optimization based on MMSE and ANOMAX}

We use the combination of the MMSE algorithm and ANOMAX algorithm [4] to obtain the optimal solution iteratively. For given $\mathbf{D}_{m}^{\prime}$, using MMSE algorithm [6] and based on the equation (12), we obtain

$$
\mathbf{D}_{m}=\sqrt{\gamma_{m}} \mathbf{C}_{m},
$$

where $\mathbf{C}_{m}=\left(\tilde{\mathbf{F}}_{m}^{H} \tilde{\mathbf{F}}_{m}+\operatorname{tr}\left(\tilde{\mathbf{R}}_{m}\right) \mathbf{I}_{K_{m}}\right)^{-1} \tilde{\mathbf{F}}_{m}^{H}, \tilde{\mathbf{F}}_{m}=$ $\overline{\mathbf{T}}_{m}^{H} \mathbf{D}_{m}^{\prime} \overline{\mathbf{T}}_{m} \mathbf{T}_{m}$ and $\gamma_{m}$ is to ensure the power constraint equation (6), i.e., $\gamma_{m}=1 / \operatorname{tr}\left(\mathbf{T}_{m} \mathbf{C}_{m} \mathbf{C}_{m}^{H}\right)$.

However, MMSE is not optimal relay precoding design strategy in the two-way relaying scenario as illustrated in [4]. Hence for given $\mathbf{D}_{m}$, we use ANOMAX algorithm to optimize the relay precoder, focusing on the effective channel gain in (12). Therefore, the optimization problem is expressed as

$$
\begin{array}{cc}
\max _{\mathbf{D}_{m}^{\prime}} & \left\|\overline{\mathbf{T}}_{m}^{H} \mathbf{D}_{m}^{\prime} \overline{\mathbf{T}}_{m} \mathbf{T}_{m} \mathbf{D}_{m}\right\|_{F}^{2} \\
\text { s.t. } & \mathbf{P}_{R_{m}}^{o w} \leq 1, m=1,2, \ldots, M .
\end{array}
$$

We assume $\mathbf{D}^{\prime}{ }_{m}=\sqrt{\gamma_{m}^{\prime}} \mathbf{C}_{m}^{\prime}$ with normalization of $\left\|\mathbf{C}_{m}^{\prime}\right\|_{F}^{2}=1$ as in [4], and to satisfy the power constraint, substituting it into the equation (10) yields

$$
\gamma_{m}^{\prime}=1 / \operatorname{tr}\left(\mathbf{C}_{m}^{\prime} \overline{\mathbf{T}}_{m}\left(\mathbf{T}_{m} \mathbf{D}_{m} \mathbf{D}_{m}^{H} \mathbf{T}_{m}^{H}+\mathbf{I}_{K_{m}}\right) \overline{\mathbf{T}}_{m}^{H} \mathbf{C}_{m}^{\prime H}\right) .
$$

Using the fact $\|\mathbf{X}\|_{F}=\|\operatorname{vec}\{\mathbf{X}\}\|_{2}$ and $\operatorname{vec}\{\mathbf{X Z Y}\}=$ $\left(\mathbf{Y}^{T} \otimes \mathbf{X}\right) \operatorname{vec}\{\mathbf{Z}\}$ as in [4], the objective function in (15) can be simplified as $\mathbf{c}^{\prime}{ }_{m}^{H} \mathbf{K}_{m}^{H} \mathbf{K}_{m} \mathbf{c}_{m}^{\prime}$, ignoring the constant factor $\gamma_{m}^{\prime}$, where $\mathbf{K}_{m}=\left(\overline{\mathbf{T}}_{m} \mathbf{T}_{m} \mathbf{D}_{m}\right)^{T} \otimes \overline{\mathbf{T}}_{m}^{H}, \mathbf{c}_{m}^{\prime}=\operatorname{vec}\left(\mathbf{C}_{m}^{\prime}\right)$ and $\operatorname{vec}(\cdot)$ operator stacks the column vectors of the matrix into a column vector. Then it can be rewritten as $\frac{\mathbf{c}_{m}^{\prime} \mathbf{K}_{m}^{H} \mathbf{K}_{m} \mathbf{c}_{m}^{\prime}}{\mathbf{c}_{m}^{\prime} \mathbf{c}^{\prime} m}$ due to the normalization. Hence, the maximum value of this function is the square of the largest singular value of $\mathbf{K}_{m}$ and

$$
\mathbf{C}_{m}^{\prime}=\operatorname{unvec}\left(\mathbf{u}_{m}\right) \text {, }
$$

where $\mathbf{u}_{m}$ is the dominant right singular vector of matrix $\mathbf{K}_{m}$ corresponding to the largest singular value and unvec $(\cdot)$ denotes the opposite operation of $\operatorname{vec}(\cdot)$. In summary, we propose a solution as in Algorithm 1

\begin{tabular}{l}
\hline Algorithm 1: based on MMSE and ANOMAX \\
\hline . Initialize power normalized matrix $\mathbf{D}_{m}$ using $(7) ;$ \\
. Repeat \\
- Compute $\mathbf{D}^{\prime}{ }_{m}$ using (16) and (17); \\
- Compute $\mathbf{D}_{m}$ using (14) ; \\
- Compute $\mathbf{D}^{\prime}{ }_{m}$ again and compute $\mathcal{I}_{m}$ using (13); \\
. Until $\mathcal{I}_{m}$ converges. \\
\hline
\end{tabular}

\section{B. Outage Optimization based on ANMwoN}

Observe that the optimal relay precoder in [4] should be scaled up to satisfy the constraint with equality while increasing the objective function, which contradicts the optimality. Here we propose the ANMwoN strategy to solve this problem.

First, we solve the optimization problem for given $\mathbf{D}_{m}^{\prime}$. To apply the ANMwoN strategy to the precoding design at the BS, we extend the beamforming structure $\mathbf{P}_{m}$ at the BS to be

$$
\overline{\mathbf{P}}_{m}=\overline{\mathbf{U}}_{m_{1}} \overline{\mathbf{D}}_{m} \overline{\mathbf{U}}_{m_{1}}^{H} \mathbf{G}_{m}^{\prime H},
$$

where $\overline{\mathbf{U}}_{m_{1}} \in \mathbb{C}^{N \times K_{m}}$ is obtained by the EVD of $\mathbf{Q}_{m}$, i.e., $\mathbf{Q}_{m}=\left[\begin{array}{ll}\overline{\mathbf{U}}_{m_{1}} & \overline{\mathbf{U}}_{m_{0}}\end{array}\right]\left[\begin{array}{cc}\overline{\boldsymbol{\Sigma}}_{m} & 0 \\ 0 & 0\end{array}\right]\left[\begin{array}{ll}\overline{\mathbf{U}}_{m_{1}} & \overline{\mathbf{U}}_{m_{0}}\end{array}\right]^{H}$. Substituting $\overline{\mathbf{P}}_{m}$ into (6) instead of $\mathbf{P}_{m}$, i.e., $\mathbf{P}_{m}^{o w}=$ $\operatorname{tr}\left\{\overline{\mathbf{D}}_{m}^{H} \overline{\mathbf{D}}_{m} \mathbb{T}_{m}^{H} \mathbb{T}_{m}\right\}$, with Hermitian matrix assumption for the initial power normalized matrix $\overline{\mathbf{D}}_{m}$, it can be expressed as

$$
\overline{\mathbf{D}}_{m}=\frac{1}{\sqrt{K_{m}}}\left(\mathbb{T}_{m}^{H} \mathbb{T}_{m}\right)^{-\frac{1}{2}}
$$

where $\mathbb{T}_{m}=\mathbf{G}_{m}^{\prime} \overline{\mathbf{U}}_{m_{1}}$, and the power constraint $\mathbf{P}_{m}^{o w}$ can be rewritten as $\mathbf{P}_{m}^{\text {ow }}=\overline{\mathbf{d}}_{m}^{H} \overline{\mathbf{R}}_{m} \overline{\mathbf{d}}_{m} \leq 1$, where $\overline{\mathbf{R}}_{m}=$ $\left(\mathbb{T}_{m}^{H} \mathbb{T}_{m}\right)^{T} \otimes \mathbf{I}_{K_{m}}$ and $\overline{\mathbf{d}}_{m}=\operatorname{vec}\left(\overline{\mathbf{D}}_{m}\right)$. Since the effective noise term is not related to $\mathbf{D}_{m}$ directly in (12), we concentrate on the effective channel gain as (15) using $\mathbb{T}_{m} \overline{\mathbf{D}}_{m} \mathbb{T}_{m}^{H}$ instead of $\mathbf{T}_{m} \mathbf{D}_{m}$ as follows

$$
\left\|\overline{\mathbf{T}}_{m}^{H} \mathbf{D}_{m}^{\prime} \overline{\mathbf{T}}_{m} \mathbb{T}_{m} \overline{\mathbf{D}}_{m} \mathbb{T}_{m}^{H}\right\|_{F}^{2} .
$$

It can be simplified as $\overline{\mathbf{d}}_{m}^{H} \overline{\mathbf{K}}_{m}^{H} \overline{\mathbf{K}}_{m} \overline{\mathbf{d}}_{m}$ where $\overline{\mathbf{K}}_{m}=$ $\left(\mathbb{T}_{m}^{H}\right)^{T} \otimes\left(\overline{\mathbf{T}}_{m}^{H} \mathbf{D}_{m}^{\prime} \overline{\mathbf{T}}_{m} \mathbb{T}_{m}^{H}\right)$. Obviously observe that the optimal vector $\tilde{\mathbf{d}}_{m}$ for the solution $\overline{\mathbf{d}}_{m}$ of such objective function must satisfy the power constraint with equality. Hence we write this optimization problem for given $\mathbf{D}_{m}^{\prime}$ as

$$
\begin{array}{cc}
\max _{\overline{\mathbf{d}}_{m}} & \overline{\mathbf{d}}_{m}^{H} \overline{\mathbf{K}}_{m}^{H} \overline{\mathbf{K}}_{m} \overline{\mathbf{d}}_{m} \\
\text { s.t. } & \overline{\mathbf{d}}_{m}^{H} \overline{\mathbf{R}}_{m} \overline{\mathbf{d}}_{m}=1 .
\end{array}
$$

Then we put power constraint into the objective function as

$$
\max _{\overline{\mathbf{d}}_{m}} \frac{\overline{\mathbf{d}}_{m}^{H} \overline{\mathbf{K}}_{m}^{H} \overline{\mathbf{K}}_{m} \overline{\mathbf{d}}_{m}}{\overline{\mathbf{d}}_{m}^{H} \overline{\mathbf{R}}_{m} \overline{\mathbf{d}}_{m}} .
$$

The problem (22) is equal to (21) since the objective function is homogeneous and any scaling in $\overline{\mathbf{d}}_{m}$ does not change the optimality. We refer to this method as ANMwoN strategy.

Proposition 1: The optimal vector $\tilde{\mathbf{d}}_{m}$ of 22 is the dominant eigenvector of $\overline{\mathbf{R}}_{m}^{-1} \overline{\mathbf{K}}_{m}^{H} \overline{\mathbf{K}}_{m}$.

Proof: Rewrite (22) as $\lambda\left(\overline{\mathbf{d}}_{m}\right)=\frac{\overline{\mathbf{d}}_{m}^{H} \overline{\mathbf{K}}_{m}^{H} \overline{\mathbf{K}}_{m} \overline{\mathbf{d}}_{m}}{\mathbf{d}_{m}^{H} \mathbf{R}_{m} \mathbf{d}_{m}}$. All local optimal vectors $\overline{\mathbf{d}}_{m}$ satisfy the first order necessary Karush-Kuhn-Tucker (KKT) condition as $\frac{\partial \lambda(\overline{\mathbf{d}})}{\partial \mathbf{d}^{*}}=0$, where $(\cdot)^{*}$ represents complex conjugation. Then we simplify KKT condition equation as $\overline{\mathbf{K}}_{m}^{H} \overline{\mathbf{K}}_{m} \overline{\mathbf{d}}_{m}=\lambda\left(\overline{\mathbf{d}}_{m}\right) \overline{\mathbf{R}}_{m} \overline{\mathbf{d}}_{m}$. Hence the optimal vector $\tilde{\mathbf{d}}_{m}$ is the dominant eigenvector of the matrix $\overline{\mathbf{R}}_{m}^{-1} \overline{\mathbf{K}}_{m}^{H} \overline{\mathbf{K}}_{m}$ due to its uniqueness.

We obtain the optimal $\overline{\mathbf{D}}_{m}$ satisfied the power constraint as

$$
\overline{\mathbf{D}}_{m}=\text { unvec }\left(\frac{1}{\tilde{\mathbf{d}}_{m}^{H} \overline{\mathbf{R}}_{m} \tilde{\mathbf{d}}_{m}} \tilde{\mathbf{d}}_{m}\right) \text {. }
$$


Second, we solve the optimization problem for given $\mathbf{D}_{m}$. Since the effective noise term is related to $\mathbf{D}^{\prime}{ }_{m}$ directly in (12), we consider another objective function including the noise term. Since the signal streams and noise streams at each user are correlated, it is difficult to analyse them. Inspired by the concept of the effective channel gain, we propose a relaxing objective function based on the ECG2ENGR as

$$
\frac{\left\|\overline{\mathbf{T}}_{m}^{H} \mathbf{D}_{m}^{\prime} \overline{\mathbf{T}}_{m} \mathbb{T}_{m} \overline{\mathbf{D}}_{m} \mathbb{T}_{m}^{H}\right\|_{F}^{2}}{\operatorname{tr}\left(\overline{\mathbf{T}}_{m}^{H} \mathbf{D}_{m}^{\prime}{ }_{m}^{\prime} \mathbf{D}_{m}^{\prime H} \overline{\mathbf{T}}_{m} \sigma_{r}^{2}+\sigma_{m}^{2} \mathbf{I}_{K_{m}}\right)} .
$$

Using ANMwoN method, we put the relay power constraint $\mathbf{P}_{R_{m}}^{o w}=\mathbf{d}_{m}^{\prime}{ }_{m}^{H} \overline{\mathbf{R}}_{m}^{\prime} \mathbf{d}_{m}^{\prime}=1$ into the second term of denominator in (24), where $\mathbf{d}_{m}^{\prime}=\operatorname{vec}\left(\mathbf{D}_{m}^{\prime}{ }_{m}\right)$ and $\overline{\mathbf{R}}_{m}^{\prime}=\left(\overline{\mathbf{T}}_{m}\left(\mathbb{T}_{m} \mathbf{D}_{m} \mathbb{T}_{m}^{H} \mathbb{T}_{m} \mathbf{D}_{m}^{H} \mathbb{T}_{m}^{H}+\mathbf{I}_{K_{m}}\right) \overline{\mathbf{T}}_{m}^{H}\right)^{T} \otimes \mathbf{I}_{K_{m}}$. Then the equation (24) can be simplified as $\frac{\mathbf{d}_{m}^{\prime H} \mathbb{K}_{m}^{H} \mathbb{K}_{m} \mathbf{d}_{m}^{\prime}}{\mathbf{d}_{m}^{\prime} \tilde{\mathbf{K}}_{m} \mathbf{d}_{m}^{\prime}}$, where $\mathbb{K}_{m}=\left(\overline{\mathbf{T}}_{m} \mathbb{T}_{m} \overline{\mathbf{D}}_{m} \mathbb{T}_{m}^{H}\right)^{T} \otimes \overline{\mathbf{T}}_{m}^{H}$ and $\tilde{\mathbf{K}}_{m}=$ $\sigma_{r}^{2}\left(\mathbf{I}_{K_{m}} \otimes\left(\overline{\mathbf{T}}_{m} \overline{\mathbf{T}}_{m}^{H}\right)\right)+\sigma_{m}^{2} \overline{\mathbf{R}}_{m}^{\prime}$. Hence the optimal matrix $\mathbf{D}_{m}^{\prime}$ can be expressed as

$$
\mathbf{D}_{m}^{\prime}=\operatorname{unvec}\left(\frac{1}{\tilde{\mathbf{d}}_{m}^{\prime H} \overline{\mathbf{R}}_{m}^{\prime} \tilde{\mathbf{d}}_{m}^{\prime}} \tilde{\mathbf{d}}_{m}^{\prime}\right),
$$

where $\tilde{\mathbf{d}}^{\prime}{ }_{m}$ is the dominant eigenvector of $\tilde{\mathbf{K}}_{m}^{-1} \mathbb{K}_{m}^{H} \mathbb{K}_{m}$. In summary, we develop an algorithm based on ANMwoN and ECG2ENGR as in Algorithm 2

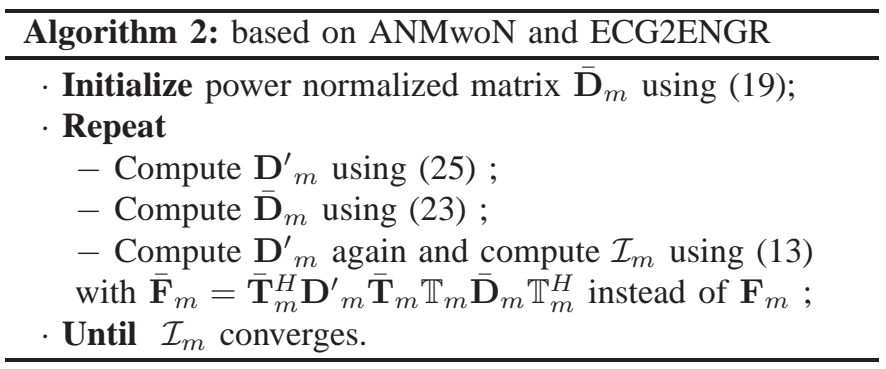

\section{Simulation Results}

In this section, we will perform simulations with the antenna configuration as $N=4, M=2$ and $K_{m}=2, m=1,2$ and the transmission data rate $R=1$ bit per channel use (BPCU). In our simulations, we set $\sigma_{m}=\sigma_{r}=\sigma_{B S}=\sigma$ such that $\mathrm{SNR}=\frac{1}{\sigma^{2}}$ and consider the Rayleigh fading channels, the components of all channel matrices follow $\mathcal{C N}(0,1)$. The proposed schemes are compared with P2PSA scheme [2], and the scheme based on the time sharing analog network coding scheme, where each user exchanges information with BS in a round robin way, which needs two time slots for one round robin.

In Fig. 11 we compare the outage probability of users for the time sharing scheme, P2PSA scheme and BSA schemes. We observe that the proposed BSA schemes can achieve better outage performance than the schemes based on time sharing and P2PSA. Since P2PSA is based on perfect diagonalization, its outage performance is the worst. Meanwhile we see that BSA schemes with iterative algorithms performance better than the BSA scheme with predefined precoders, and the BSA scheme with algorithm 2 is the best among all BSA schemes since it is based on ANMwoN and ECG2ENGR. In Fig. 2

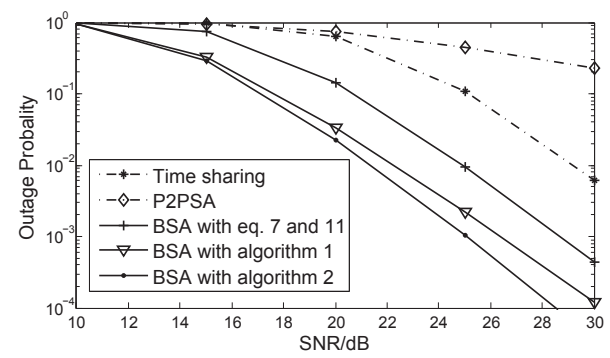

Fig. 1. Outage Probability for users, $\operatorname{Pro}\left(I_{m}<2 K_{m} R\right), R=1 \mathrm{BPCU}$, $N=4, M=2$ and $K_{m}=2, m=1,2$.

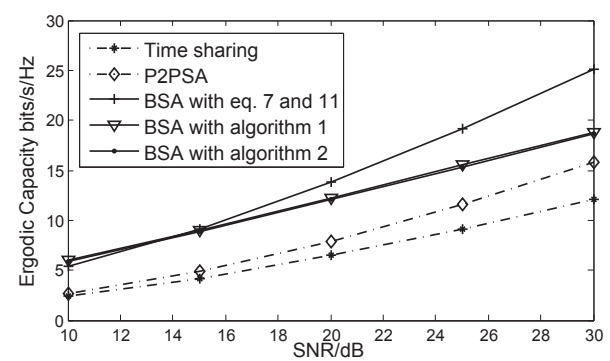

Fig. 2. Ergodic Capacity, $N=4, M=2$ and $K_{m}=2, m=1,2$.

we compare their ergodic capacity performance. As shown from the figure, the schemes based on BSA and P2PSA are superior to the time sharing scheme because the latter needs many time slots to accomplish the whole transmission. BSA schemes yield a rates improvement compared with the P2PSA scheme, due to the larger aligned space. Note that the BSA scheme with predefined precoders yields higher rates than BSA schemes with algorithm 1 and 2, because these two algorithms are designed to outage optimization for users, not optimal for maximizing the sum-rate of the whole system and they tend to concentrate most of the energy on the dominant singular value, not beneficial to the sum-rate over high SNRs.

\section{CONCLUSION}

We have developed a coordinated beamforming scheme to null the inter-user interference for a MIMO cellular network using a approach of BSA. Moreover, we have also proposed two algorithms to jointly optimize the beamforming matrices at the relay and BS for outage optimization. Simulation results have been provided for performance evaluation.

\section{REFERENCES}

[1] C. Esli and A. Wittneben, "Multiuser MIMO Two-way Relaying for Cellular Communications," in Proc. IEEE PIMRC 2008, Cannes, France, Sep. 2008.

[2] Z. Ding, I. Krikidis, J. Thompson and K. Leung, "Physical Layer Network Coding and Precoding for the Two-Way Relay Channel in Cellular Systems", IEEE Trans. Signal Process., vol. 59, no. 2, pp. 696-712, Feb. 2011.

[3] Q. H. Spencer, A. L. Swindlehurst, and M. Haardt, "Zero-forcing methods for downlink spatial multiplexing in multiuser mimo channels," IEEE Trans. Signal Process. , vol. 52, no. 2, pp. 461-471, Feb. 2004.

[4] F. Roemer and M. Haardt, "Algebraic Norm-Maximizing (ANOMAX) transmit strategy for Two-Way relaying with MIMO amplify and forward relays," IEEE Signal Process. Lett., vol. 16, Oct. 2009.

[5] H. Xin, Y. Peng, C. Wang, Y. Yang and W. Wang, "Coordinated Eigen Beamforming for Multi-Pair MIMO Two-Way Relay Network," in Proc. IEEE GLOBECOM 2011, Houston, TX, Dec. 2011.

[6] M. Joham, W. Utschick, and J. A. Nossek, "Linear Transmit Processing in MIMO Communication Systems," IEEE Trans. Signal Process., vol. 53, no. 8, pp. 2700-2712, Aug. 2005. 